\title{
PERIODIC SOLUTIONS OF AUTONOMOUS FUNCTIONAL DIFFERENTIAL EQUATIONS
}

\author{
BY ROGER D. NUSSBAUM ${ }^{1}$
}

Communicated by Philip Hartman, November 20, 1972

\begin{abstract}
The purpose of this note is to indicate some applications of a new fixed point theorem to the question of periodic solutions of nonlinear autonomous functional differential equations. The techniques developed give the standard periodicity examples in the literature and some new results, notably for the neutral case, which do not seem accessible by previous methods.
\end{abstract}

1. If $X$ is a Banach space and $A$ is a bounded subset of $X$, define $\gamma(A)$, the measure of noncompactness of $A$, to be $\inf \{d>0: A$ has a finite covering by sets of diameter less than $d\}$. This is a notion due to $C$. Kuratowski [13]. G. Darbo observed [4] that if $\overline{\mathrm{co}}(A)$ denotes the convex closure of a set $A$ and if $A+B=\{a+b: a \in A, b \in B\}$ for sets $A$ and $B$, then (1) $\gamma(\overline{\mathrm{co}}(A))=\gamma(A)$ and $(2) \gamma(A+B) \leqq \gamma(A)+\gamma(B)$. It is trivially true that (3) $\gamma(A \cup B)=\max \{\gamma(A), \gamma(B)\}$.

For applications it is sometimes convenient to generalize the above idea slightly. If $\mu$ is a function which assigns to each bounded subset $A$ of $X$ a real number $\mu(A)$, we say that $\mu$ is a generalized measure of noncompactness if $\mu$ satisfies properties (1), (2) and (3) above and if there exist positive constants $m$ and $M$ such that $m \mu(A) \leqq \gamma(A) \leqq M \mu(A)$ for every set $A \subset X$. If $J$ is a closed bounded interval of $R$ and $C^{1}\left(J, R^{n}\right)$ denotes the Banach space of continuously differentiable maps from $J$ to $\boldsymbol{R}^{n}$ with any of the standard norms, then if

$$
\mu(A)=\lim _{\delta \rightarrow 0 ; \delta>0}\left(\sup \left\{\left|x^{\prime}(t)-x^{\prime}(s)\right|: x \in A, t, s \in J,|t-s|<\delta\right\}\right),
$$

$\mu$ is an example of a generalized measure of noncompactness on $C^{1}\left(J, R^{n}\right)$.

If $U$ is a subset of a Banach space $X, f: U \rightarrow X$ is a continuous map, and $\mu$ is a generalized measure of noncompactness, then we shall say that $f$ is a $k$-set-contraction with respect to $\mu$ if for every bounded set $A \subset U$, $f(A)$ is bounded and $\mu(f(A)) \leqq k \mu(A)$. If $G$ is a closed, convex subset of $X, U$ is a bounded open subset of $G$, and $f: \bar{U} \rightarrow G$ is a $k$-set-contraction with respect to $\mu, k<1$, then if $f(x) \neq x$ for $x \in \bar{U}-U$, there is an integer defined, called the fixed point index of $f$ on $U$ and written $i_{G}(f, U)$. Details are given in [15], where the fixed point index is actually defined for a larger class of maps which are defined on open subsets of certain metric

AMS (MOS) subject classifications (1970). Primary 34K15, 47H10.

Key words and phrases. Periodic solutions, functional differential equations, fixed point theorems, fixed point index.

${ }^{1}$ Partially supported by NSF GP-20228 and, a Rutgers Research Council Faculty Fellowship. 
ANR's. The fixed point index can be thought of as an algebraic count of the number of fixed points of $f$ in $U$. If $G=X$ and $f$ is compact, $i_{G}(f, U)=$ $\operatorname{deg}(I-f, U, 0)$, the Leray-Schauder degree of $I-f$ on $U$.

Before stating our main fixed point theorem we need one more definition. Suppose that $C$ is a topological space, $x_{0} \in C, W$ is an open neighborhood of $x_{0}$, and $f: W-\left\{x_{0}\right\} \rightarrow C$ is a continuous map. Then we shall say that $x_{0}$ is an "ejective point of $f$ " if there exists an open neighborhood $U$ of $x_{0}$ such that for every $x \in U-\left\{x_{0}\right\}$, there exists an integer $m=m(x)$ such that $f^{m}(x)$ is defined and $f^{m}(x) \notin U$. This definition is a slight generalization of one given by $\mathrm{F}$. E. Browder in [2], where he assumed that $f$ is defined and continuous at $x_{0}$ and $f\left(x_{0}\right)=x_{0}$. However, for some applications $f$ cannot be defined continuously at $x_{0}$.

THEOREM 1. Let $G$ be a closed, bounded, convex and infinite dimensional subset of a Banach space $X, x_{0} \in G$, and $f: G-\left\{x_{0}\right\} \rightarrow G$ a continuous map such that $x_{0}$ is an ejective point of $f$. Assume that $f$ is a $k$-set-contraction with respect to $\mu, k<1$, where $\mu$ is a generalized measure of noncompactness on $X$. Then if $U$ is an open neighborhood of $x_{0}$ in $G$ such that $f$ has no fixed points in $\bar{U}-\left\{x_{0}\right\}, i_{G}(f, G-\bar{U})=1$ and $f$ has a fixed point in $G-\bar{U}$. If $G$ is finite dimensional (not equal to a point) and $x_{0}$ is an extreme point of $G$, the same conclusion holds.

Theorem 1 is not hard to obtain by use of some ideas from [2] and standard techniques in asymptotic fixed point theory. Its significance lies in its direct applicability to a number of concrete analytic problems. We should mention, however, that there are situations (see Theorem 3 below) in which Theorem 1 seems inapplicable and other asymptotic fixed point theorems are needed.

COROLLARY 1. Let $G$ be a closed, bounded, convex and infinite dimensional subset of a Banach space $X, \mu$ a generalized measure of noncompactness on $X$ and $f: G \rightarrow G$ a k-set-contraction with respect to $\mu, k<1$. Then $f$ has a fixed point which is not an ejective point of $f$. If $x_{0}$ is an ejective fixed point of $f$ and $U$ is an open neighborhood of $x_{0}$ in $G$ such that $f(x) \neq x$ for $x \in \bar{U}-\left\{x_{0}\right\}$, then $i_{G}(f, U)=0$.

Corollary 1 is a direct generalization of Browder's work in [1], [2] and Jones's fixed point theorems in [11].

COROLlaRY 2. Let $G$ be a closed, convex and infinite dimensional subset of a Banach space $X$ such that $0 \in G$. If $\mu$ is a generalized measure of noncompactness on $X, U=\{x \in G:\|x\|<R\}$, and $f: \bar{U}-\{0\} \rightarrow G$ is a $k$-set-contraction with respect to $\mu, k<1$, such that 0 is an ejective point of $f$ and $f(x) \neq t x$ for $t \geqq 1$ and $\|x\|=R$, then if $W=\{x \in G: r<\|x\|<R\}$ and $f(x) \neq x$ for $0<\|x\| \leqq r, i_{G}(f, W)=1$ and $f$ has a fixed point in $W$. 
2. Using Theorem 1 and its corollaries we have obtained the periodicity results given by G. S. Jones in [9], [11] and R. B. Grafton in [5], [6]. Rather than repeat these results we mention some simple new examples which appear inaccessible by previous methods. All of the examples given below admit more or less mechanical generalization, but for ease of exposition we restrict ourselves to the cases below. In all of the examples we give, the fixed point index of an appropriately defined map equals one; in a future paper we hope to give examples of a completely different type for which a fixed point index equals minus one.

Our first example is a generalized van der Pol equation of the type considered by Grafton in [5], [6]. The case $k<0$ below partially answers a question raised by Grafton in [6].

THEOREM 2. The equation

$$
x^{\prime \prime}(t)-\varepsilon x^{\prime}(t)\left[1-x^{2}(t)\right]+x(t-r)-k x(t)=0
$$

has a nontrivial periodic solution of period greater than $2 r$ if $\varepsilon>0, r>0$ and $-k_{0}<k<1$, where $k_{0}=\min \left(\varepsilon / r, 2 / r^{2}\right)$.

The following equation was mentioned by Halanay and Yorke in [7]. Its analysis involves some nonstandard techniques.

THEOREM 3. The equation $x^{\prime}(t)=\alpha x(t-1-|x(t)|)\left(1-x^{2}(t)\right)$ has a nontrivial periodic solution of period greater than 2 if $\alpha>\pi / 2$.

THEOREM 4. The neutral functional differential equation

$$
x^{\prime}(t)=\left[-\alpha x(t-1)+(k / m+1)(d / d t) x^{m+1}(t-1)\right]\left[1-x^{2}(t)\right]
$$

has a nontrivial periodic solution of period greater than 2 if $\alpha>\pi / 2$, $m \geqq 1$ and $|k| \leqq[(m+1) / 4][1+2 / m-1]^{m-1 / 2}\left(|k| \leqq \frac{1}{2}\right.$ if $\left.m=1\right)$.

Since the equation $x^{\prime}(t)=-\alpha x(t-1)\left(1-x^{2}(t)\right)$ is perhaps the best understood of all nonlinear autonomous FDE's (with regard to periodic solutions), the neutral FDE in Theorem 4 is one of the simplest nonlinear, neutral FDE's imaginable, but even in this case there are unanswered questions and Theorem 4 is not best possible.

ACKNOWLEDGEMENTS. I would like to thank my colleague, Don Orth, who very kindly ran computer numerical simulations of the equations in Theorems 2-4. These simulations suggested improvements in the original versions of Theorems 2-4.

\section{REFERENCES}

1. F. E. Browder, Another generalization of the Schauder fixed point theorem, Duke Math. J. 32 (1965), 399-406. MR 34 \# 3567.

2. 1 A further generalization of the Schauder fixed point theorem, Duke Math. J. 32 (1965), 575-578. MR 37 \# 3568. 
3. F. E. Browder, Asymptotic fixed point theorems, Math. Ann. 185 (1970), 38-60. MR 73 \#1165.

4. G. Darbo, Punti uniti in trasformazioni a condiminio non compatto, Rend. Sem. Mat. Univ. Padova 24 (1955), 84-92. MR 16, \#1140.

5. R. B. Grafton, A periodicity theorem for autonomous functional differential equations, J. Differential Equations 6 (1969), 87-109. MR 39 \#4500.

6, - Periodic solutions of certain Lienard equations with delay, J. Differential Equations 11 (1972), 519-527.

7. A. Halanay and J. Yorke, Some new results and problems in the theory of differentialdelay equations, SIAM Rev. 13 (1971), 55-80. MR 44 \# 1899.

8. J. Hale, Functional differential equations, Appl. Math. Sciences, vol. 3, SpringerVerlag, New York, 1971.

9. G. S. Jones, The existence of periodic solutions of $f^{\prime}(x)=-\alpha f(x-1)[1+f(x)]$,

J. Math. Anal. Appl. 5(1962), 435-450. MR 25 \# 5234.

10. - Asymptotic fixed point theorems and periodic solutions of functional differential equations, Contributions to Differential Equations 2 (1963), 385-405. MR 28 \# 1361.

11. - Periodic motions in Banach space and applications to functional differential equations, Contributions to Differential Equations 3(1964), 75-106. MR 29 \#342.

12. J. Kaplan and J. Yorke, On the stability of a periodic solution of a differential-delay equation, SIAM J. Math. Anal. (to appear).

13. C. Kuratowski, Sur les espaces complets, Fund. Math. 15 (1930), 301-309.

14. R. D. Nussbaum, Asymptotic fixed point theorems for local condensing maps, Math. Ann. 191 (1971), 181-195.

15. , The fixed point index for local condensing maps, Ann. Mat. Pura Appl. 89 (1971), 217-258.

16. Some fixed point theorems, Bull. Amer. Math. Soc. 77 (1971), 360-365. MR 77 \#2112.

17. - , Some asymptotic fixed point theorems, Trans. Amer. Math. Soc. 171 (1972), 349-375.

18. E. M. Wright, $A$ non-linear difference-differential equation, J. Reine Angew Math. 194 (1955), 66-87. MR 17, 272.

19. R. D. Nussbaum, Periodic solutions of some nonlinear autonomous functional differential equations, Ann. Mat. Pura Appl. (to appear).

Department of Mathematics, Rutgers University, New Brunswick, New Jersey 08903

Current address: Institute for Advanced Study, Princeton, New Jersey 08540 